\title{
BIRTH AND DEATH PROCESSES WITH ABSORPTION
}

\author{
MOURAD E. H. ISMAIL \\ University of South Florida \\ Department of Mathematics \\ Tampa, FL 33620, U.S.A. \\ JEAN LETESSIER \\ GALLIANO VALENT \\ Laboratoire de Physique Théorique et Hautes Energies \\ Unité associée au CNRS UA 280 \\ Université Paris 7, FRANCE
}

(Received May 8, 1991)

\begin{abstract}
Spectral measures and transition probabilities of birth and death processes with $\lambda_{0}=\mu_{0}=0$ are obtained as limite when $\lambda_{0}-0^{+}$of the corresponding quantities. In particular the case of finite population is discussed in full detail. Pure birth and death processes are used to derive an inequality for Dirichlet polynomials.
\end{abstract}

KEY WORDS AND PHRASES. Markov process, and inequality for Dirichlet polynomials. 1991 AMS SUBJECT CLASSIFICATION CODES. 60J, 60J20.

\section{Introduction.}

A birth and death process is a stationary Markov process, has state space $\{0,1, \ldots\}$ and the transition probabilities $p_{m, n}(t)$ (the probabilty that the system takes time $t$ to go from state $m$ to state $n$ ) satisfy the forward equation

$$
\frac{d}{d t} p_{m, n}(t)=\lambda_{n-1} p_{m, n-1}(t)+\mu_{n+1} p_{m, n+1}(t)-\left(\lambda_{n}+\mu_{n}\right) p_{m, n}(t)
$$

and the backward equation

$$
\frac{d}{d t} p_{m, n}(t)=\lambda_{m} p_{m+1, n}(t)+\mu_{m} p_{m-1, n}(t)-\left(\lambda_{m}+\mu_{m}\right) p_{m, n}(t) .
$$

Usually the birth rates $\left\{\lambda_{n}\right\}_{0}^{\infty}$ and the death rates $\left\{\mu_{n}\right\}_{0}^{\infty}$ in (1) and (2) are assumed to satisfy

$$
\mu_{0} \geq 0, \lambda_{n}>0 \text { and } \mu_{n+1}>0, n \geq 0 .
$$

Karlin and McGregor [7] have shown that the $p_{m, n}(t)$ 's admit the integral representation

$$
p_{m, n}(t)=\frac{\lambda_{0} \lambda_{1} \ldots \lambda_{n-1}}{\mu_{1} \ldots \mu_{n}} \int_{0^{-}}^{\infty} Q_{m}(x) Q_{n}(x) d \mu(x),
$$

where $\left\{Q_{n}(x)\right\}_{0}^{\infty}$ are polynomials orthogonal with respect to the spectral measure $d \mu(x)$. Here $d \mu(x)$ is a positive probability measure and the $Q_{n}$ 's are generated by 


$$
\begin{aligned}
-x Q_{n}(. x) & =\lambda_{n} Q_{n+1}(x)+\mu_{n} Q_{n-1}(x)-\left(\lambda_{n}+\mu_{n}\right) Q_{n}(x) . \quad \| \geq 1, \\
Q_{0}(x) & =1, \quad Q_{1}(x)=\left(\lambda_{0}+\mu_{0}-x\right) / \lambda_{0},
\end{aligned}
$$

and satisfy the orthogonality relation

$$
\int_{0^{-}}^{\lambda} Q_{n}(x) Q_{m}(x) d \mu(x)=\frac{\mu_{1} \ldots \mu_{n}}{\lambda_{0} \ldots \lambda_{n-1}} \delta_{m, n} .
$$

If probability is to be conserved, i.e. the process is honest [7]. [15]. 1he antrictum

$$
\mu_{0}=0
$$

must be imposed. The interested reader may consult our recent survey article [6] for details.

The purpose of this paper is to consider absorbing processes with $\lambda_{0}=0$ and to derive an inequality for Dirichlet polynomials. We consider processes with $\lambda_{0}=0$ as limiting cases $\lambda_{0} \rightarrow 0^{+}$ of birth and death processes with $\lambda_{0}>0$. The general theory for this procedure is explained in Section 2 for a finite state space and outlined in Section 3 for an infinite state spacc. In Sicction 1 we illustrate the procedure of Sections 2 and 3 by applying it to the cases of linear birth and death processes. The dual case where we start with processes having $\lambda_{0}=0, \mu_{0}$ nonvanishing, taking the limit $\mu_{0} \rightarrow 0^{+}$may be handled in the same way using the result obtained in [12].

Linear birth and death processes have bcen studied extensively. An exellent reference to consult is [8]. Birth and death processes with finite state space are also called truncated processes, [3]. Applications of truncated birth and death processes are in [9] and [10] for exemple. Karlin and Tavaré [11] considered birth and death processes with killing. For such process

$$
\begin{aligned}
p_{\imath, j}(t) & =\lambda_{t} t+o(t), \quad j=i+1, \\
& =\mu_{\imath} t+o(t), \quad j=i-1, \\
& =1-\left(\lambda_{\imath}+\mu_{\imath}+\gamma_{\imath}\right) t+o(t), \quad j=\imath,
\end{aligned}
$$

as $t \rightarrow 0^{+}$, where $\gamma_{2}$ is the rate of killing or absorption at state $i$. The results of Sections 2 and 3 can be extended to birth and death processes with killing in a straightforward way. The only difference is that $x=0$ may no longer support a discrete mass even if the state space is finite.

This paper concludes with a Section 5 where we show how pure birth (or pure death) processes imply the curious inequality

$$
1 \geq \sum_{j=m}^{n} \lambda_{j} e^{-t \lambda,} \prod_{\substack{m \leq l \leq n \\ l \neq j}}\left(1-\lambda_{j} / \lambda_{l}\right)^{-1} \geq 0, t>0, n \geq m,
$$

if the $\lambda_{j}$ 's are distinct. The cases when some of the $\lambda_{j}$ 's are equal are limiting cases of the cases with distinct $\lambda$,'s. In Section 5 we also discuss Dirichlet series representations for $p_{m, n}(t)$ and $\sum_{n=m}^{\infty} p_{m, n}(t)$ for a pure birth process. As an example we consider the case $\lambda_{n}=\alpha\left(n^{2}+\beta n+\gamma\right)$.

\section{$2 \quad$ Finite state spaces.}

Recall that (7) is assumed throughout this section. From (5) we see that $\lambda_{0} Q_{1}(x) \rightarrow-x$ and $\lambda_{0} Q_{2}(x) \rightarrow-x\left(\lambda_{1}+\mu_{1}-x\right) / \lambda_{1}$ as $\lambda_{0} \rightarrow 0$. By induction we see from (5) that 


$$
\lambda_{u} Q_{n}(x) \rightarrow-x \phi_{n-1}(x) . n>0, \text { as } \lambda_{0} \rightarrow 0
$$

where $\phi_{n}(x)$ has precise degrec 11 , and

$$
\begin{gathered}
\varphi_{u}(x)=1, \phi_{1}(x)=\left(\lambda_{1}+\mu_{1}-x\right) / \lambda_{1}, \\
\lambda_{n} \phi_{n}(x)=\left(\lambda_{n}+\mu_{n}-x\right) \phi_{n-1}(x)-\mu_{n} \phi_{n-2}(x), n>1 .
\end{gathered}
$$

Furthermore one can show by induction that $Q_{n}(0)=1$ for all $n, n \geq 0$.

In many interesting cases the $\lambda_{n}$ 's depend on a parameter $\alpha$ and $\lambda_{0} \rightarrow 0$ when $\alpha$ tends to a critical value $\alpha_{0}$, but as $\alpha \rightarrow \alpha_{0}$ the $\lambda_{n}$ 's and $\mu_{n}$ 's, $n>0$, have non zero limits. This is the case for example when $\lambda_{n}=n+\alpha+1, \mu_{n}=n$ and $\alpha \rightarrow-1$.

It is clear that the $\phi_{n}$ 's are birth and death process polynomials associated with birth rates $\left\{\lambda_{n}\right\}_{1}^{\infty}$ and death rates $\left\{\mu_{n}\right\}_{1}^{\infty}$.

In this section we treat the case of finite state space (finite population) because it is easier and makes certain features more transparent. Let the state space be $\{0,1, \ldots, N\}$. Define numerator polynomials, [1], [17], $Q_{n}^{*}(x)$ as the solution of the recursion in (5) with the initial conditions

$$
Q_{0}^{*}(x)=0, \quad Q_{1}^{*}(x)=-1 / \lambda_{0}
$$

It is more convenient in spectral analysis to use the monic polynomials

$$
P_{n}(x)=(-1)^{n} \lambda_{0} \ldots \lambda_{n-1} Q_{n}(x), \quad P_{n}^{*}(x)=(-1)^{n} \lambda_{0} \ldots \lambda_{n-1} Q_{n}^{*}(x)
$$

It is easy to see that

$$
P_{n}(x)=\left|\begin{array}{cccccc}
x-\lambda_{0} & \mu_{1} & 0 & \ldots & 0 & 0 \\
\lambda_{0} & x-\lambda_{1}-\mu_{1} & \mu_{2} & \ldots & 0 & 0 \\
0 & \lambda_{1} & x-\lambda_{2}-\mu_{2} & \ddots & 0 & 0 \\
\vdots & \vdots & \ddots & \ddots & \ddots & \ldots \\
0 & 0 & 0 & \ddots & \ddots & \mu_{n-1} \\
0 & 0 & 0 & \vdots & \lambda_{n-2} & x-\lambda_{n-1}-\mu_{n-1}
\end{array}\right|, n>0,
$$

Observe that the determinent representations in (14) and (15) are meaningful even if some of $\lambda_{n}$ 's and $\mu_{n}$ 's vanish. Note that

$$
\phi_{n}(x)=(-1)^{n} \lambda_{1} \lambda_{2} \ldots \lambda_{n} P_{n+1}^{*}(x)
$$


We now assume $\lambda_{N}=0$, which forces the state space to be $\{0,1, \ldots, N\}$.

The Stieltjes transform of $d / \mu$. . the spectral measure of the finite process, is giren by

$$
\frac{P_{x+1}^{*}(x)}{P_{i+1}(x)}=\int_{-x}^{x} \frac{d \mu_{x}(t)}{x-t}
$$

The zeros of $P_{N+1}(x)$ are all real and simple and interlace with the zeros of $P_{N+1}^{*}(x)$. [17]. Furthermore

$$
\lim _{x \rightarrow \infty} x \frac{P_{N+1}^{*}(x)}{P_{N+1}(x)}=1 .
$$

Thus $d_{\mu_{N}}(t)$ has masses $A_{0}, A_{1} \ldots, A_{N}$ at the zeros $x_{0}<x_{1}<\ldots<x_{N}$ of $P_{N+1}(x)$ and

$$
\frac{P_{N+1}^{*}(x)}{P_{N+1}(x)}=\sum_{k=0}^{N} \frac{A_{k}}{x-x_{k}} . \quad \sum_{k=0}^{N} A_{k}=1, \quad A_{k}>0,0 \leq k \leq N .
$$

The sum of the rows in the determinant $P_{N+1}(x)$ is $x$, hence $P_{N+1}(0)=0$. The remaining zeros of $P_{N+1}(x)$ are positive. Thus $x_{0}=0$ and (19) becomes

$$
\frac{P_{N+1}^{*}(x)}{P_{N+1}(x)}=\frac{A_{0}}{x}+\sum_{k=1}^{N} \frac{A_{k}}{x-x_{k}} . \quad \sum_{k=0}^{N} A_{k}=1 . \quad A_{k}>0,0 \leq k \leq . N .
$$

As $\lambda_{0} \rightarrow 0 . P_{N+1}(x) \rightarrow x P_{N+1}^{*}(x)$, hence $A_{0} \rightarrow 1$. Therefore $A_{k} \rightarrow 0,1 \leq k \leq N$ and the spectral measure converges to a single point mass at $x=0$. Later we will see that this is a general phenomenon when the state space is the nonnegative integers and the moment problem is determined.

Recall that for $N>2, A_{k}$ is also given by

$$
A_{k}=\left[1+\sum_{n=1}^{N} P_{n}^{2}\left(x_{k}\right) / \prod_{j=1}^{n}\left(\lambda_{j-1} \mu_{\jmath}\right)\right]^{-1} .
$$

This shows that for $k>0, \lambda_{0}>0$,

$$
A_{k}=\lambda_{0} \mu_{1}\left[\lambda_{0} \mu_{1}+\sum_{n=1}^{N} P_{n}^{2}\left(x_{k}\right) / \prod_{\jmath=2}^{n}\left(\lambda_{\jmath-1} \mu_{\jmath}\right)\right]^{-1} .
$$

Therefore

$$
A_{k} / \lambda_{0} \rightarrow \mu_{1}\left[P_{1}^{2}\left(\tilde{x}_{k}\right)+\sum_{n=2}^{N-1} P_{n}^{2}\left(\tilde{x}_{k}\right) / \prod_{\jmath=2}^{n}\left(\lambda_{\jmath-1} \mu_{\jmath}\right)\right]^{-1}, k>0, \quad \tilde{x}_{k}=\lim _{\lambda_{0} \rightarrow 0^{+}} x_{k} .
$$

Observe that $P_{n}\left(\tilde{x}_{k}\right)=\tilde{x}_{k} P_{n}^{*}\left(\tilde{x}_{k}\right), 1 \leq k \leq N$ and $\left\{P_{n}^{*}(x)\right\}_{1}^{N}$, or $\left\{\phi_{n}(x)\right\}_{0}^{N-1}$, is a finite family of polynomials orthogonal with respect to a discrete (positive) probability measure $d \mu_{N}^{(1)}(x)$ such that

$$
\int_{-\infty}^{\infty} \frac{d \mu_{N}^{(1)}(x)}{x-t}=\sum_{k=1}^{N} \frac{B_{k}}{x-\tilde{x}_{k}}, \quad B_{k}=\left[1+\sum_{n=2}^{N-1}\left[P_{n}^{*}\left(x_{k}\right)\right]^{2} / \prod_{\jmath=1}^{n}\left(\mu_{\jmath+1} \lambda_{\jmath}\right)\right]^{-1} .
$$

Thus we proved:

Theorem 1 As $\lambda_{0} \rightarrow 0^{+}$, the spectral measure $d \mu(x)$ degenerates to a single unit mass supported at $x=0$. Furthermore the $\phi_{n}$ 's are orthogonal with respect to a discrete probability measure $d \mu_{N}^{(1)}(x)$ supported on a finite subset of $(0, \infty)$ and 


$$
\frac{x^{2} d \mu(x)}{\lambda_{0} \mu_{1}} \rightarrow d \mu_{N}^{(1)}(x)
$$

Theorem 1 and equation (1) furnish complete information about the limits of the transition probabilities $p_{m, n}(t)$ as $\lambda_{0} \rightarrow 0^{+}$. Indeed by writing $\int_{0^{-}}^{\infty} f(x) d \mu(x)$ as $f(0) A_{1}+\int_{0^{+}}^{\infty}[f(x)-f(0)] d \mu(x)$ we obtain for $m, n>0$

$$
p_{m, n}(t)=\frac{\lambda_{1} \ldots \lambda_{n-1}}{\mu_{2} \ldots \mu_{n}}\left[\frac{\lambda_{0}}{\mu_{1}} A_{v} Q_{n}(0) Q_{m}(0)+\int_{0^{+}}^{\infty}{ }^{-x t} \lambda_{v} Q_{n}(x) \lambda_{0} Q_{m}(x) \frac{d \mu(. x)}{\lambda_{0} \mu_{1}}\right],
$$

hence

$$
\lim _{\lambda_{0} \rightarrow 0^{+}} p_{m, n}(t)=\frac{\lambda_{1} \ldots \lambda_{n-1}}{\mu_{2} \ldots \mu_{n}} \int_{0}^{\infty} \epsilon^{-x t} \phi_{n-1}(x) \phi_{m-1}(x) d \mu^{(1)}(x) . \quad m, n>0 .
$$

Clearly $p_{0,0}(t) \rightarrow 1$ as $\lambda_{0} \rightarrow 0$.

We also have, for $m>0$,

$$
p_{m, 0}(t)=\int_{0^{-}}^{\infty} e^{-x t} Q_{m}(x) d \mu(x)=A_{0}+\int_{0^{+}}^{\infty} e^{-x t} \lambda_{0} Q_{m}(x) \frac{d \mu(x)}{\lambda_{0}}
$$

so that

$$
\lim _{\lambda_{0} \rightarrow 0^{+}} p_{m, 0}(t)=1+\mu_{1} \int_{0}^{\infty} \frac{e^{-x t}}{x} \phi_{m-1}(x) d \mu^{(1)}(x), \quad m>0 .
$$

Similarly for $n>0$,

$$
\begin{aligned}
p_{0, n}(t) & =\frac{\lambda_{0} \ldots \lambda_{n-1}}{\mu_{1} \ldots \mu_{n}} \int_{0^{-}}^{\infty} e^{-x t} Q_{n}(x) d \mu(x) \\
& =\frac{\lambda_{1} \ldots \lambda_{n-1}}{\mu_{1} \ldots \mu_{n}}\left[\lambda_{0} A_{0}+\int_{0^{+}}^{\infty} e^{-x t} \lambda_{0} Q_{n}(x) d \mu(x)\right]
\end{aligned}
$$

and

$$
p_{0, n}(t) \rightarrow 0, \quad m>0, \text { as } \lambda_{0} \rightarrow 0^{+}
$$

Therefore

$$
\lim _{\lambda_{0} \rightarrow 0^{+}} p_{0, n}(t)=\delta_{n, 0}
$$

\section{$3 \quad$ Infinite state spaces.}

We now extend the results of Section 2 to the case when the state space is the nonnegative integers. We assume that the spectral measure is essentially unique, that is the moment problem associated with the polynomials $\left\{Q_{n}(x)\right\}$ is determined. We refer the interested reader to [17] for criteria for the determinancy of the moment problem.

If $m>0, n>0$ then, by letting $\lambda_{0} \rightarrow 0^{+}$in (6), we see that $\lim _{\lambda_{0} \rightarrow 0^{+}} \frac{x^{2}}{\lambda_{0} \mu_{1}} d \mu(x)=d \mu^{(1)}(x)$ holds in the sense that

$$
\int_{0}^{\infty} f(x) \frac{x^{2}}{\lambda_{0} \mu_{1}} d \mu(x) \rightarrow \int_{0}^{\infty} f(x) d \mu^{(1)}(x) \text { as } \lambda_{0} \rightarrow 0^{+},
$$

for any polynomial $f(x)$ independent of $\lambda_{0}$. This also shows that $\left\{\phi_{n}\right\}_{0}^{\infty}$ are orthogonal with respect to $d \mu^{(1)}(x)$ and 


$$
\int_{0}^{\lambda} \phi_{n}(x) \varphi_{m}(. r) d \mu^{(1)}(. x)=\frac{\mu_{2} \mu_{3} \ldots \mu_{n-1}}{\lambda_{1} \ldots \lambda_{n}} \delta_{m, n}, m, n \geq 0 .
$$

A more delicate argument shows that the limit relation in (32) holds if $f$ depends on $\lambda_{v}$ but $f(x) c^{l x}$ converges to a polynomial as $\lambda_{0} \rightarrow 0^{+}$. for all $t>0, x \geq 0$. This shows that

$$
\lim _{\lambda_{0} \rightarrow 0^{+}} p_{m n}(t)=\frac{\lambda_{1} \ldots \lambda_{n-1}}{\mu_{2} \ldots \mu_{n}} \int_{0}^{\infty}{ }^{-t x} \phi_{m-1}(x) \phi_{n-1}(. x) d \mu^{(1)}(x), m . n>0 .
$$

as expected. To see that $d \mu^{(1)}(x)$ has total mass equal to 1 we express $x^{2}$ in terms of $Q_{0}(x), Q_{1}(. x)$ and $Q_{2}(x)$ as

$$
\frac{x^{2}}{\lambda_{0}}=\lambda_{1} Q_{2}(x)-\left(\lambda_{0}+\mu_{1}+\lambda_{1}\right) Q_{1}(x)+\left(\lambda_{0}+\mu_{0}\right) Q_{0}(x) .
$$

Then integrate both sides with respect to $d \mu(x) / \mu_{1}$ on $[0, \infty)$ and apply (6) to get

$$
\int_{0}^{\infty} \frac{x^{2}}{\lambda_{0} \mu_{1}} d \mu(x) \rightarrow 1 \text { as } \lambda_{0} \rightarrow 0^{+}
$$

The first moment of $d \mu$ is

$$
\int_{0^{-}}^{\infty} x d \mu(x)=\int_{0^{-}}^{\infty} x Q_{0}(x) d \mu(x)=\int_{0^{-}}^{\infty}\left[\lambda_{0} Q_{0}(x)-\lambda_{0} Q_{1}(x)\right] d \mu(x)=\lambda_{0} .
$$

If $f(x)$ is a polynomial of degree at least 2 then

$$
\int_{0^{-}}^{\infty} f(x) d \mu(x)=\int_{0^{-}}^{\infty} \frac{\left[f(x)-f(0)-x f^{\prime}(0)\right]}{x^{2}} x^{2} d \mu(x)+f(0)+\int_{0^{-}}^{\infty} x d \mu(x)
$$

If $f(x)$ is independent of $\lambda_{0}$ the above identity (32) and (37), yields

$$
\lim _{\lambda_{0} \rightarrow 0^{+}} \int_{0^{-}}^{\infty} f(x) d \mu(x)=f(0) .
$$

This shows that as $\lambda_{0} \rightarrow 0^{+}, d \mu(x)$ degenerates to having a unit mass at $x=0$. In fact we proved:

Theorem 2 Let the moment problem corresponding to the polynomials $Q_{n}(x)$ be determined. Then as $\lambda_{0} \rightarrow 0^{+}, d \mu(x)$ degenerates to a singleton of unit mass at $x=0$ but

$$
\lim _{\lambda_{0} \rightarrow 0^{+}} \frac{x^{2} d \mu(x)}{\lambda_{0} \mu_{1}}=d \mu^{(1)}(x),
$$

where $d \mu^{(1)}(x)$ is a probability measure supported on $(0, \infty)$. Furthermore the $\phi_{n}$ 's are orthogonal with respect to $d \mu^{(1)}(x)$.

Next consider the cases $m>0, n=0$ and $m=0, n \geq 0$. Here we need to distinguish between the case when $\mu$ is continuous at $x=0$ and the case when $\mu$ has a jump at $x=0$. First assume

$$
\mu\left(0^{+}\right)-\mu\left(0^{-}\right)=A_{0}\left(\lambda_{0}\right)>0
$$

for $\lambda_{0}$ in a neighborhood of $\lambda_{0}=0$, so that

$$
\begin{aligned}
p_{m, 0}(t) & =\int_{0^{-}}^{\infty} e^{-t x} Q_{m}(x) d \mu(x) \\
& =A_{0}\left(\lambda_{0}\right)+\int_{0^{+}}^{\infty} e^{-t x}\left[Q_{m}(x)-Q_{m}(0)\right] d \mu(x)
\end{aligned}
$$

Clearly $A_{0}\left(\lambda_{0}\right) \rightarrow 1$ as $\lambda_{0} \rightarrow 0^{+}$and 


$$
\lim _{l_{0} \rightarrow 0^{+}} \mu_{m .0}(t)=1+\mu_{1} \int_{0}^{x},^{-t x} \phi_{m-1}(x) \cdot x^{-1} d \mu^{(1)}(x) . \quad m>0
$$

if $\int_{0}^{\alpha} e^{-t x} x^{-1} d \mu^{(1)}(x)$ exists. On the other hand if $\mu$ is continuous at 0 for $\lambda_{0}$ in a meighlourlexod of $\lambda_{0}=0$ then

$$
\lim _{l_{0} \rightarrow 0^{+}} p_{m, 0}(t)=\mu_{1} \int_{0}^{\infty} c^{-t x} \phi_{m-1}(x) x^{-1} d \mu^{(1)}(x), \quad m>0
$$

In the case $m=0, n \geq 0$, since $\lambda_{0}=0$, the backward equation (1) shows that $p_{0, n}(t)$ is independent of 1 . The forward equation (2) and simple induction proves that

$$
p_{0, n}(t) \rightarrow \delta_{n, 0}, \text { when } \lambda_{0}=0^{+}
$$

\section{Examples.}

In this section we shall consider tow linear birth and death processes with

$$
\begin{aligned}
& \lambda_{n}=n+\alpha+1, \mu_{n}=n, \\
& \lambda_{n}=c(n+\beta), \mu_{n}=n .
\end{aligned}
$$

There is no loss of generality in assuming $\mu_{n}=n$ since we require $\mu_{0}=0$.

We shall also discuss a birth and death process with rates

$$
\lambda_{n}=n+\alpha+1, \mu_{n+1}=n+c+1, n \geq 0, \mu_{0}=0 .
$$

This later case is more complicated but the analysis is of some interest.

The case of constant birth and death rates involves Chebyshev polynomials and we leave it as an exercise for the interested reader.

\subsection{The process (45).}

In this case

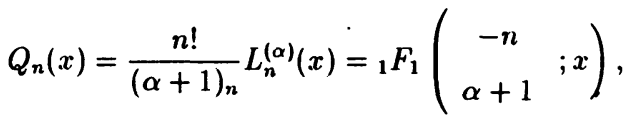

where $\left\{L_{n}^{(\alpha)}(x)\right\}_{0}^{\infty}$ are the Laguerre polynomials [14]. Hence the $Q_{n}$ 's satisfy the orthogonality relation

$$
\int_{0}^{\infty} Q_{n}(x) Q_{m}(x) \frac{x^{\alpha}}{\Gamma(\alpha+1)} d x=\frac{n !}{(\alpha+1)_{n}} \delta_{m, n}
$$

It is easy to see that

$$
\begin{aligned}
\phi_{n}(x) & =\lim _{\alpha \rightarrow-1}-\frac{(\alpha+1)}{x} Q_{n+1}(x)=\lim _{\alpha \rightarrow-1} \sum_{k=0}^{n+1} \frac{(-n-1)_{k}}{k !(\alpha+1)_{k}}(-\alpha-1) x^{k-1} \\
& =-\sum_{k=1}^{n+1} \frac{(-n-1)_{k}}{k !(k-1) !} x^{k-1}=(n+1) x L_{n}^{(1)}(x) .
\end{aligned}
$$

Now (14) holds since

$$
\frac{x^{2} x^{\alpha} e^{-x}}{(\alpha+1) \Gamma(\alpha+1)} \rightarrow-x e^{-x} \text { as } \alpha \rightarrow-1
$$

Thus

$$
d \mu^{(1)}(x)=x e^{-x} d x
$$


Therefore as $\alpha \rightarrow-1$ we obtain

$$
\begin{aligned}
& p_{m, n}(t) \rightarrow m \frac{(\alpha+2)_{n-1}}{(n-1) !} \int_{0}^{\infty} e^{-t x} L_{m-1}^{(1)}(x) L_{n-1}^{(1)} x e^{-x} d x, m, n>0 \\
& p_{m, 0}(t) \rightarrow m \int_{0}^{\infty} e^{-t x} L_{m-1}^{(1)}(x) e^{-x} d x, m>0 \\
& p_{0, n}(t) \rightarrow \delta_{0, n}, n \geq 0
\end{aligned}
$$

\subsection{The process (46).}

In the case under consideration, [2, VI.3.2],

$$
Q_{n}(x)=m_{n}(x /(1-c) ; \beta, c) /(\beta)_{n},
$$

where the $m_{n}$ 's are Meixner polynomials. The orthogonality relation of the Meixner polynomials, [2, VI.3.4], takes the form

$$
\sum_{k=0}^{\infty} Q_{n}(k(1-c)) Q_{j}(k(1-c)) c^{k} \frac{(\beta)_{k}}{k !}(1-c)^{\beta}=\frac{c^{-n} n !}{(\beta)_{n}} \delta_{n, j} .
$$

Now $\lambda_{0} \rightarrow 0^{+}$in two different ways, either by letting $\beta \rightarrow 0^{+}$or by letting $c \rightarrow 0$. We shall treat only the former case because the latter case becomes a case of a pure death process.

Thus as $\lambda_{0} \rightarrow 0^{+}, x^{2} \frac{d \mu(x)}{\lambda_{0} \mu_{1}}$ converge to $d \mu^{(1)}(x)$ where $\mu^{(1)}(x)$ is a jump function. $\mu^{(1)}(-\infty)=0$ and $\mu^{(1)}(x)$ has jump $k c^{k-1}(1-c)^{2}$ at $x_{k}=(1-c) k, k=1,2, \ldots$ Clearly $\mu^{(1)}(\infty)=1$, as expected. Observe that in this case $\frac{d \mu(x)}{\lambda_{0} \mu_{1}}$ blows up at $x=0$ as $\lambda_{0} \rightarrow 0^{+}$, while $x^{2} \frac{d \mu(x)}{\lambda_{0} \mu_{1}}$ converges when $\lambda_{0} \rightarrow 0^{+}$.

It is easy to see that

$$
\phi_{n}(x)=m_{n}\left(\frac{x-1}{1-c} ; 2, c\right) /(n+1) !
$$

hence one can use in (34), (42), (44) to compute the transition probabilities.

\subsection{The process (47).}

In this case the $Q_{n}$ 's are related to a family of associated Laguerre polynomials $\left\{\mathcal{L}_{n}^{(\alpha)}(x ; c)\right\}$ in [5] by

$$
Q_{n}(x)=\frac{(c)_{n}}{(\alpha+c+1)_{n}} \mathcal{L}_{n}^{(\alpha)}(x ; c)
$$

It was shown in [5] that

$$
\frac{d \mu(x)}{d x}=x^{\alpha} e^{-x} \mid \Psi\left(c,-\operatorname{alpha} ;\left.x e^{-i x}\right|^{-2} /\{\Gamma(c+1) \Gamma(1+c+\alpha)\},\right.
$$

where $\Psi$ is a Tricomi psi function [4, Chapter 6]. We let $\lambda_{0} \rightarrow 0^{+}$by letting $\alpha \rightarrow-1-c$. Thus in the limit we have

$$
\frac{x^{2}}{\lambda_{0} \mu_{1}} \frac{d \mu(x)}{d x} \rightarrow \frac{x^{1-c} e^{-x}}{\Gamma(c+2)}\left|\Psi\left(c, c+1 ; x e^{-i \pi}\right)\right|^{-2} .
$$

But the basic integral representation $[4,6.5 .2]$ shows that $\Psi(a, a+1 ; x)=x^{-a}$, when Re $a>0$. Therefore

$$
\frac{x^{2}}{\lambda_{0} \mu_{1}} \frac{d \mu(x)}{d x} \rightarrow \frac{x^{1+c} e^{-x}}{\Gamma(c+2)},
$$

i.e. the $\phi_{n}$ 's are essentially Laguerre polynomials with parameter $c$. This can be also shown directly from the recurrence relation. 
Finally we note that the limiting case $\beta \rightarrow-c$ in the birth and death process,

$$
\lambda_{n}=c(n+B+c) . \quad \mu_{n+1}=n+c, n \geq 0, \quad \mu_{0}=0 .
$$

can be similary handled and the $\phi_{n}$ 's are Meixner polynomials.

\section{Pure birth processes and an inequality.}

We apply the methode of separation of variables to find the transition probabilities. The result is not new but the proof is of some interest because it also applies to general birth and death processes as pointed out in our joint work with D. Masson [6]. We shall consider first the case of a finite state space $\{0,1, \ldots, N\}$ and assume that $\lambda_{0}, \lambda_{1}, \ldots, \lambda_{N}$ are distinct.

$$
p_{m, n}(t)=f(t) F_{n} Q_{m}
$$

and substitute in (1) and (2) to get

$$
\frac{f^{\prime}(t)}{f(t)}=\left[\lambda_{n-1} F_{n-1}-\lambda_{n} F_{n}\right] / F_{n}=\left[\lambda_{m} Q_{m+1}-\lambda_{m} Q_{m}\right] / Q_{m}
$$

Thus each side in the above equality must be a constant. Set

$$
f^{\prime}(t) / f(t)=-x
$$

Since the probabilities $p_{m, n}(t)$ always lie in $(0,1)$ then $x \geq 0$. This shows that

$$
Q_{m+1}=\left(1-x / \lambda_{m}\right) Q_{m}, \quad F_{n-1}=\left(\lambda_{n}-x\right) F_{n} / \lambda_{n-1}
$$

hence

$$
Q_{m}=Q_{0} \prod_{j=0}^{m-1}(1-x / \lambda,)
$$

and similarly

$$
F_{n}=\frac{\lambda_{N}}{\lambda_{n}} F_{N} \prod_{j=n+1}^{N}\left(1-x / \lambda_{j}\right)
$$

One can incorporate $Q_{0}$ and $F_{N}$ in the separation constants and find

$$
p_{m, n}(t)=\frac{\lambda_{N}}{\lambda_{n}} \int_{0}^{\infty} e^{-t x} \prod_{j=n+1}^{N}\left(1-x / \lambda_{j}\right) \prod_{i=1}^{m-1}\left(1-x / \lambda_{i}\right) d \mu(x),
$$

where $d \mu(x)$ is a measure which depends on $N$. In a pure birth process we must have $p_{m, n}(t)=0$ if $n<m$, and $p_{m, n}(t) \rightarrow \delta_{m, n}$ as $t \rightarrow 0^{+}$.

The first restriction implies

$$
\prod_{j=n+1}^{N}(1-x / \lambda,) \prod_{i=0}^{m-1}\left(1-x / \lambda_{i}\right) d \mu(x)=0, m>n,
$$

a.e., hence we may choose $d \mu(x)$ to be supported at $\left\{\lambda_{0}, \lambda_{1}, \ldots, \lambda_{N}\right\}$. The second restriction is

$$
\frac{\lambda_{N}}{\lambda_{n}} \sum_{l=0}^{N} c_{l} \prod_{j=n+1}^{N}\left(1-\lambda_{l} / \lambda_{j}\right) \prod_{i=0}^{m-1}\left(1-\lambda_{l} / \lambda_{i}\right)=\delta_{m, n} .
$$


where $c_{l}$ is the (possibly signed) mass at $\lambda_{l}$. The solution of the above system is

$$
c_{l}=\left(\lambda_{l} / \lambda_{N}\right) \prod_{\substack{0 \leq i \leq N \\ \mathfrak{l} \neq l}}\left(1-\lambda_{l} / \lambda_{l}\right)^{-1}
$$

therefore

$$
p_{m, n}(t)=\frac{1}{\lambda_{n}} \sum_{j=m}^{n} \lambda_{j} e^{-t \lambda,} \prod_{\substack{m \leq \leq l \leq n \\ l \neq j}}\left(1-\lambda_{J} / \lambda_{l}\right)^{-1}, n \geq m
$$

This proves the inequality ( 8 ).

Observe that the restriction $N<\infty$ may now be removed from (70) provided that the series on the right side converges. A sufficient condition for this is that $\lambda_{j+1}-\lambda_{j}$ is bounded below by a positive number for sufficiently large $j$.

Using a completely different technique, McCarthy et al. [13], proved that a nontrivial Dirichlet polynomial $\sum_{k=1}^{n} a_{k} x^{\lambda_{k}}$, where $\lambda_{k} \in R$ and the $\lambda$ 's are distinct, has at most $n-1$ zeros in $R^{+}$. This shows that the series appering in (8) may have at most $n-m$ zeros when $t<0$.

When

$$
\lambda_{n}=\alpha\left(n^{2}+\beta n+\gamma\right)=\alpha(n+a)(n+b),
$$

on can compute explicitly the products in (70). In this case

$$
\prod_{\substack{m \leq l \leq n \\ l \neq j}}\left\{\left(\lambda_{l}-\lambda_{j}\right) / \alpha\right\}=\frac{(-1)^{j-m}(j-m) !(n-j) ! \Gamma(n+j+\beta+1)}{(2 j+\beta) \Gamma(m+j+\beta)}
$$

and

$$
p_{m, n}(t)=\sum_{j=m}^{n} \frac{(m+a)_{n-m}(m+b)_{n-m}(2 j+\beta)(\beta)_{m+\jmath}}{(j-m) !(n-j) !(\beta)_{n+\jmath+1}}(-1)^{\jmath-m} e^{-\lambda, t}
$$

Clearly

$$
\begin{gathered}
\sum_{n=m}^{\infty} p_{m, n}(t)=\sum_{n, j=0}^{\infty} \frac{(-1)^{j}(m+a)_{n+j}(m+b)_{n+j}(\beta)_{2 m+j}}{j ! n !(\beta)_{n+2 m+2 j+1}}(2 j+2 m+\beta) e^{-\lambda_{m+j} t} \\
=\sum_{j=0}^{\infty} \frac{(-1)^{\jmath}(m+a)_{j}(m+b)_{j}(\beta)_{2 m+j}}{j !(\beta)_{2 m+2 j+1}}(2 j+2 m+\beta) e^{-\lambda_{m+j} t} \\
\times{ }_{2} F_{1}\left(\begin{array}{c}
a+m+j, b+m+j \\
\beta+2 j+2 m+1
\end{array} ; 1\right) .
\end{gathered}
$$

A simplification leads to

$$
\sum_{n=m}^{\infty} p_{m, n}(t)=\sum_{j=0}^{\infty} \frac{(-1)^{j}(m+a)_{j}(m+b)_{j}}{j !(\beta+2 m+j)_{j}} e^{-\lambda_{m+t_{2}} F_{1}}\left(\begin{array}{c}
a+m+j, b+m+j \\
\beta+2 j+2 m+1
\end{array} ; 1\right) .
$$

The above formula is a corrected version of a formula obtained by Roehner [16] using generating functions. On can use Gauss's theorem, Rainville [14], 


$$
{ }_{2} F_{1}\left(\begin{array}{cc}
a, b & ; 1 \\
c &
\end{array}\right)=\frac{\Gamma(c) \Gamma(c-a-b)}{\Gamma(c-a) \Gamma(c-b)}
$$

to get

$$
\sum_{n=m}^{\infty} p_{m, n}(t)=\frac{\Gamma(\beta)}{\Gamma(m+a) \Gamma(m+b)} \sum_{j=0}^{\infty} \frac{(-1)^{\jmath}(2 j+2 m+\beta)(\beta)_{2 m+j}}{(a+m+j)(b+m+j) j !} e^{-\lambda_{m+\jmath^{t}}}
$$

When $a=b$ and $\beta$ is an integer the above sum can be expressed in terms of the Jacobi theta function $\theta_{1}$, its derivatives and its integral. Roehner simplified the expression $\sum_{n=m}^{\infty} p_{m, n}(t)$ only when $m=0, a=b=1 / 2$ (so $\beta=1$ ). Snyder [18] considered the case $a=b=1$ (so $\beta=2$ ).

ACKNOWLEDGEMENT. We thank David Masson and Bertrand Roehner for many stimulating discussions during the preparation of this note. Research partially supported by grant DMS8814026 from the National Science Foundation.

\section{References}

[1] R. Askey and M. E. H. Ismail, Recurrence relations, continued fractions and orthogonal polynomials, Memoirs Amer. Math. Soc. 300 (1984).

[2] T.S. Chihara, "An Introduction to Orthogonal Polynomials", Gordon and Breach, New York, 1978.

[3] E. A. van Doorn, On the time dependent behaviour of the truncated birth-death process, Stochastic Processes and their Appl. 11 (1981), 261-271.

[4] A. Erdélyi, W. Magnus, F. Oberhettinger and F. G. Tricomi, "Higher Transcendental Functions", volume 1, McGraw-Hill, New York, 1953.

[5] M. E. H. Ismail, J. Letessier and G. Valent, Linear birth and death models and associated Laguerre polynomials, J. Approximation Theory 56 (1988), 337-348.

[6] M. E. H. Ismail, J. Letessier, D. Masson and G. Valent, Birth and death processes and orthogonal polynomials, in "Orthogonal Polynomials: Theory and Practice", P. Nevai, ed., NATO ASI series C, 294 (1990), 229-255.

[7] S. Karlin and J. McGregor, The differential equations of birth and death processes and the Stieltjes moment problem, Transactions Amer. Math. Soc. 85 (1958), 489-546.

[8] S. Karlin and J. McGregor, Linear growth, birth and death processes, J. Math. and Mech. (now Indiana Math. J.) 7 (1958), 643-662. 
[9] S. Karlin and J. McGregor, On a genetic model of Moran, Proc. Cambridge Philos. Sioc. 58 (1962), 299-311.

[10] S. Karlin and J. McGregor, Ehrenfest urn models, J. Appl. Prob. 2 (1965), 352-376.

[11] S. Karlin and S. Tavaré, Linear birth and death processes with killing, J. Appl. Prob. 19 (1982), 477-487.

[12] J. Letessier and G. Valent, Dual birth and death processes and orthogonal polynomials, SIAM J. Appl. Math. 46 (1986), 393-405.

[13] P. C. McCarthy, J. E. Sayre and B. L. R. Shawyer, Generalised Legendre polynonials, to appear.

[14] E. D. Rainville, "Special Functions", Chelsea, New York, 1971.

[15] G. E. H. Reuter, Denumerable Markov processes and associated semigroupes on l, Acta Math. 97 (1957), 1-46.

[16] B. Roehner, Stochastic versus deterministic non-linear growth processes, Int. J. Systems Sci. 17 (1986),1153-1162.

[17] J. A. Shohat and J. D. Tamarkin, "The Problem of Moments", Mathematical Surveys, volume 1, American Mathematical Society, Providence, Rhode Island, 1950.

[18] D. L. Snyder, "Random Point Processes", Wiley, New York, 1975. 


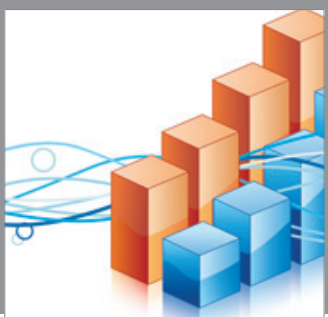

Advances in

Operations Research

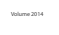

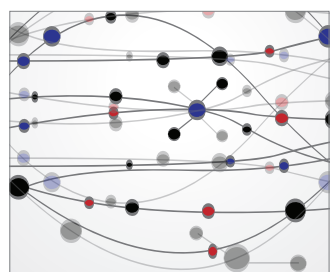

\section{The Scientific} World Journal
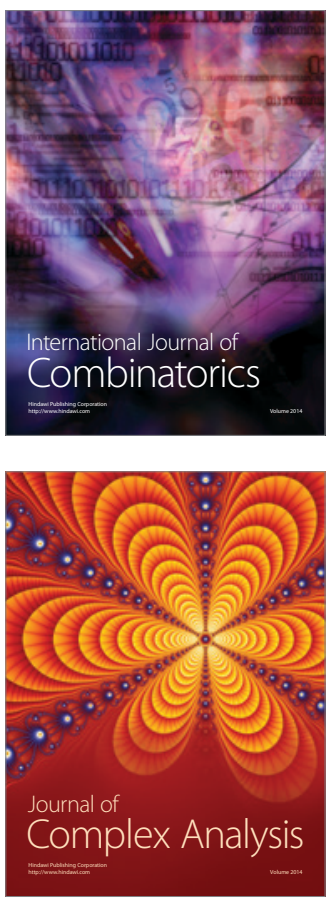

International Journal of

Mathematics and

Mathematical

Sciences
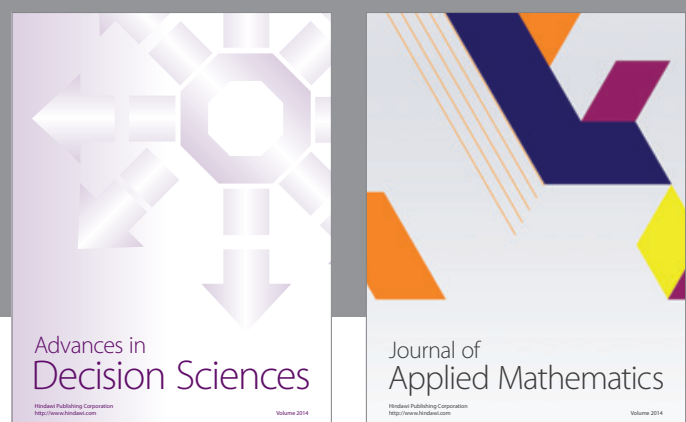

Journal of

Applied Mathematics
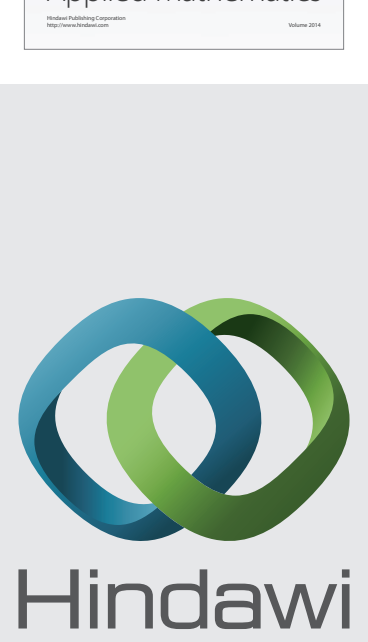

Submit your manuscripts at http://www.hindawi.com
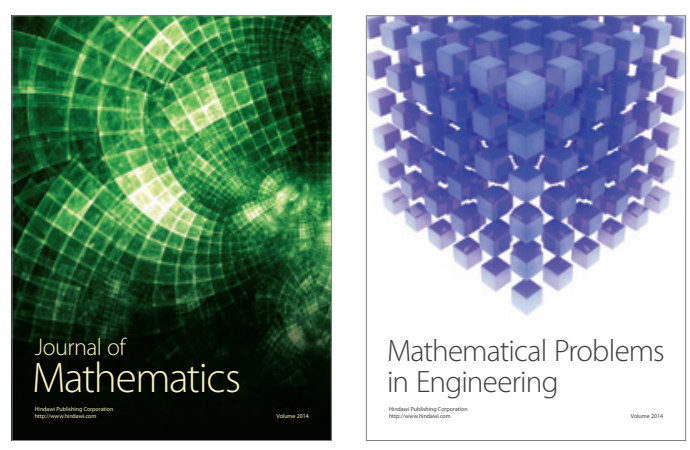

Mathematical Problems in Engineering
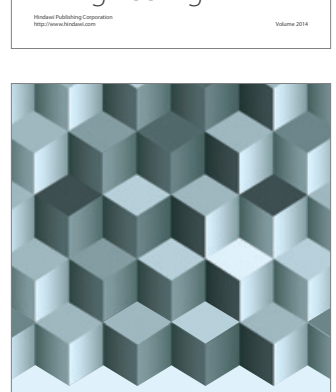

Journal of

Function Spaces
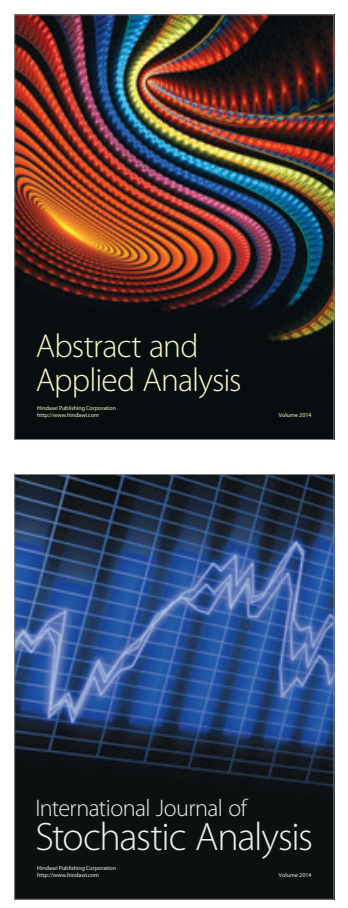

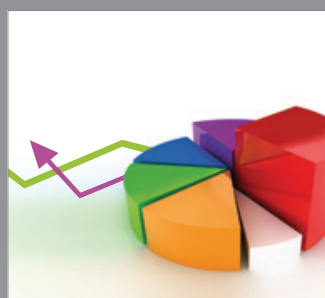

ournal of

Probability and Statistics

Promensencen
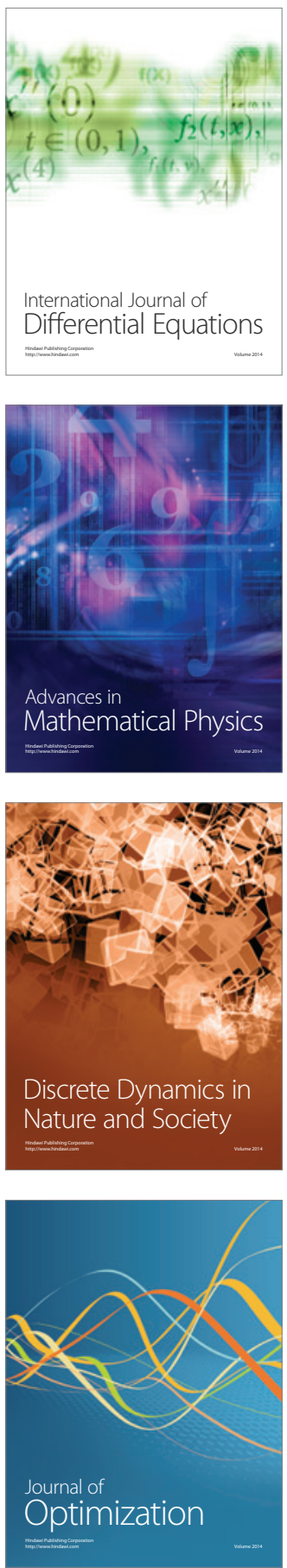